\title{
Observation of the plasma channel dynamics and Coulomb explosion in the interaction of a high-intensity laser pulse with a He gas jet
}

\author{
G. S. Sarkisov, ${ }^{\text {a) }}$, V. Yu. Bychenkov, and V. T. Tikhonchuk \\ P. N. Lebedev Physics Institute, Russian Academy of Sciences, 117924 Moscow, Russia
}

\author{
A. Maksimchuk, S. Y. Chen, R. Wagner, G. Mourou, and D. \\ Umstadter \\ Center for Ultrafast Optical Science, University of Michigan, Ann Arbor, MI 48109-2099, \\ USA
}

(Submitted 11 November 1997)

Pis'ma Zh. Éksp. Teor. Fiz. 66, No. 12, 787-792 (25 December 1997)

\begin{abstract}
We report the first interferometric observations of the dynamics of electron-ion cavitation of relativistically self-focused intense $4 \mathrm{TW}$, $400 \mathrm{fs}$ laser pulse in a He gas jet. The electron density in a channel 1 $\mathrm{mm}$ long and $30 \mu \mathrm{m}$ in diameter drops by a factor of approximately 10 from the maximum value of $\sim 8 \times 10^{19} \mathrm{~cm}^{-3}$. A high radial velocity of the plasma expansion, $\sim 3.8 \times 10^{8} \mathrm{~cm} / \mathrm{s}$, corresponding to an ion energy of about $300 \mathrm{keV}$, is observed. The total energy of fast ions is estimated to be $6 \%$ of the laser pulse energy. The high-velocity radial plasma expulsion is explained by a charge separation due to the strong ponderomotive force. This experiment demonstrates a new possibility for direct transmission of a significant portion of the energy of a laser pulse to ions. (C) 1997 American Institute of Physics.

[S0021-3640(97)00824-4]
\end{abstract}

PACS numbers: 52.50.Jm, 52.20.Hv, 42.65.Jx

A number of proposed applications of ultrahigh-intensity, short laser pulses implies laser guiding for distances much longer than the Rayleigh length. Guiding of intense laser pulses in underdense plasmas due to the relativistic self-focusing was first reported in Ref. 1 and then studied in detail in Refs. 2-6. However, the dynamics of plasma channel at high laser intensities and phenomena associated with its expansion have not yet been addressed. This letter presents new experimental results on the dynamics of the plasma channel produced by an ultrahigh-intensity, short laser pulse and offers a theoretical interpretation for these results.

The experiment was performed using the $10 \mathrm{TW}$ Ti:sapphire-Nd:glass laser system based on chirped-pulse amplification, ${ }^{7}$ developed at the Center for Ultrafast Optical Science, University of Michigan. The laser operates at the wavelength $\lambda=1.053 \mu \mathrm{m}$ and produces 3-J, 400-fs FWHM pulses with an intensity contrast of $10^{5}$. The laser beam, 50 $\mathrm{mm}$ in diameter, was focused with an off-axis parabolic mirror $(f / 3.3, f=16.5 \mathrm{~cm})$ to a $10 \mu \mathrm{m}$ spot with a vacuum intensity $6 \times 10^{18} \mathrm{~W} / \mathrm{cm}^{2}$. Laser beam was focused in a high-back-pressure $(7 \mathrm{MPa}) \mathrm{He}$ gas jet expanding through a nozzle $1 \mathrm{~mm}$ in diameter. 
The optimal conditions for beam guiding correspond to focusing of the laser on the jet edge, with a He atom density $n_{\mathrm{He}} \simeq 3 \times 10^{19} \mathrm{~cm}^{-3}$. The jet thickness was about $1 \mathrm{~mm}$. A two-channel optical setup has been used for simultaneous recording of interferometric and shadow plasma images. The probe beam was split from the main beam, propagated through an adjustable optical delay, and was incident on a plasma in the direction perpendicular to the interaction beam. The plasma was imaged using a single spherical lens with angular aperture $\pm 7^{\circ}$ on two cooled 12-bit CCD cameras. The spatial and temporal resolution was $10 \mu \mathrm{m}$ and $400 \mathrm{fs}$, respectively. For the electron density measurements the air-wedge shearing interferometer ${ }^{8}$ was used.

The plasma evolution was investigated in the time interval from -2 to $+55 \mathrm{ps}$. As $t=0$ we take the time of arrival of the maximum laser intensity in the focal plane $z=0$. The first signs of gas ionization were observed at $t=-2 \mathrm{ps}$. This indicates the laser intensity is above the He ionization threshold, $\sim 10^{15} \mathrm{~W} / \mathrm{cm}^{2}$, at that time. ${ }^{9} \mathrm{We}$ observed a fast gas ionization in the cone of laser convergence, which is $8.6^{\circ}$. It propagates along the laser axis approximately at the speed of light until it reaches the rear side of the jet. On the time of ionization (from -2 to +3 ps) the front part of interferogram is blurred because of the fringe motion during the exposure time. The first signatures of plasma channel formation (fringe bending on the laser axis) were observed at $t=0 \mathrm{ps}$. The channel length increases with subluminal velocity up to $1000 \mu \mathrm{m}$.

The interferometric and shadow plasma images taken at $t=20 \mathrm{ps}$ are shown in Fig. 1 for a laser energy of $1.7 \mathrm{~J}(4.3 \mathrm{TW})$. The local opposite displacement of the interference fringes in a narrow axial region in the interferogram indicates a decrease of the phase shift in this region and hence a decrease of the electron density. This region is manifested in the shadow image by a bright narrow line, which is due to refraction of the probe beam on the high radial electron density gradient. The two-dimensional reconstruction of the electron density profile for $t=35 \mathrm{ps}$ (assuming the axial symmetry of a plasma) for the same laser conditions is presented in Fig. 2 a. The maximum electron density is $7.6 \times 10^{19}$ $\mathrm{cm}^{-3}$ at a radius of $\simeq 20 \mu \mathrm{m}$ and the depth of the plasma channel is up to $80-90 \%$. The accuracy of measurement of the channel depth is limited by the Abel inversion procedure.

The dynamics of electron density profile near the focal plane $(z=100 \mu \mathrm{m})$ is shown in Fig. 2b. The electron density gradient at the channel walls reaches the value of $5 \times 10^{22}$ $\mathrm{cm}^{-4}$ at a time of $7 \mathrm{ps}$ and remains practically the same out to $55 \mathrm{ps}$. The evolution of the lineal electron density (number of electrons per unit length $N_{e}=2 \pi \int r d r n_{e}(r)$ ) at $z=100 \mu \mathrm{m}$ and the evolution of the mean electron density in the same cross section are presented in Fig. 3a. After the initial phase of fast ionization (from -2 to $0 \mathrm{ps}$ ) the number of electrons remains constant (from 0 to $9 \mathrm{ps}$ ), and then ionization starts again. At the same time the average electron density starts to decrease. These features are in agreement with the temporal behavior of the plasma radius at $z=100 \mu \mathrm{m}$ (shown in Fig. $3 \mathrm{~b}$ ). It remains approximately the same from 0 to $9 \mathrm{ps}$ then the plasma begins to expand radially with almost constant velocity. If we define the plasma edge as a region where the electron density equals $5 \times 10^{18} \mathrm{~cm}^{-3}$ (17\% ionization), then the expansion velocity equals $3.8 \times 10^{8} \mathrm{~cm} / \mathrm{s}$ (curve 1 in Fig. $3 \mathrm{~b}$ ). The region of higher degree of ionization, $1.5 \times 10^{19} \mathrm{~cm}^{-3}\left(50 \%\right.$ ionization), expands at a lower velocity $\sim 2.5 \times 10^{8} \mathrm{~cm} / \mathrm{s}$ (curve 2 in Fig. 3b).

We attribute the plasma expansion to ionization of the ambient gas by fast ions 


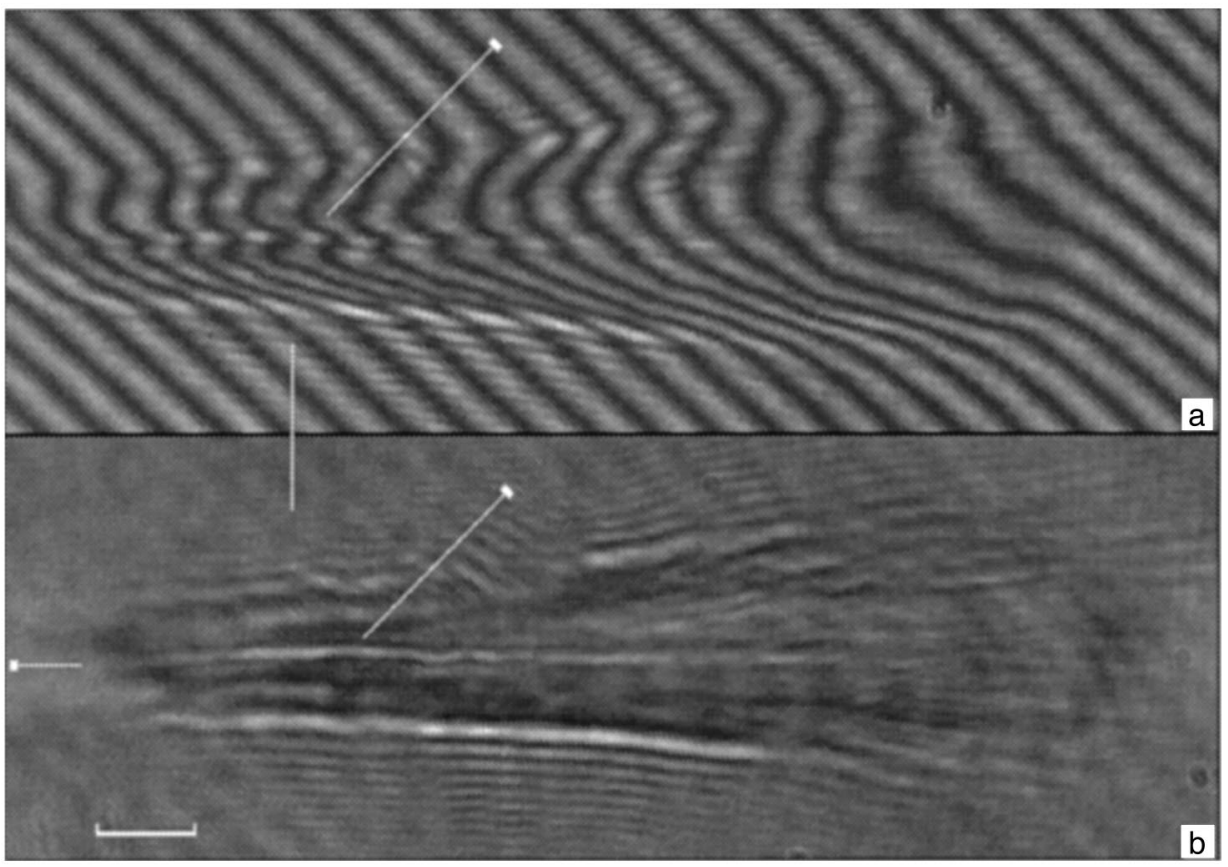

FIG. 1. Interferometric (a) and shadow (b) images of a plasma at time $20 \mathrm{ps}$ after focusing $1.7 \mathrm{~J}, 4.3 \mathrm{TW}$ laser pulse. The $100 \mu \mathrm{m}$ spatial scale is shown in left corner. The vertical line in the shadow image marks the position of focal plane. Arrows indicate the plasma channel. The horizontal arrow indicates the direction of the interaction laser beam.

expelled from the channel. Then the expansion of plasma edge (with 17\% ionization) can be related to the propagation of fast ions with an energy of about $300 \mathrm{keV}$, while the expansion of the main plasma volume (with 50\% ionization) corresponds to $130 \mathrm{keV}$ ions. This assumption of collisional ionization of the gas by fast ions also explains the $\sim 10$ ps delay time, which is the time needed for fast ions to penetrate through the laser-ionized plasma volume and reach the ambient gas. (The velocity of the plasma profile at a given density is lower than the actual ion velocity on account of the radial ion expansion and the decrease of the ion flux. However, we neglect this difference in present paper and, therefore, underestimate the ion energy.)

The channel diameter (Fig. 3b) also changes with time. Up to time $t=9$ ps we do not observe a significant variation of channel diameter $D \sim 10-15 \mu \mathrm{m}$, which is at the limit of our spatial resolution. At $t>9$ ps the channel diameter increases to $25-35 \mu \mathrm{m}$ at a velocity of about $6 \times 10^{7} \mathrm{~cm} / \mathrm{s}$.

The initial laser beam channeling can be attributed to the effect of relativistic selffocusing of intense laser pulse. ${ }^{1}$ The critical power for relativistic self-focusing $P_{c}=17 n_{c} / n_{e} \mathrm{GW}$ (where $n_{e}$ is the electron density and $n_{c}$ is the critical density) corresponds under our conditions to $280 \mathrm{GW}$, which is more than 10 times lower than the actual laser power. Therefore we speculate that a substantial part of the laser power is trapped in a narrow channel near the laser axis. The amount of trapped power depends on 


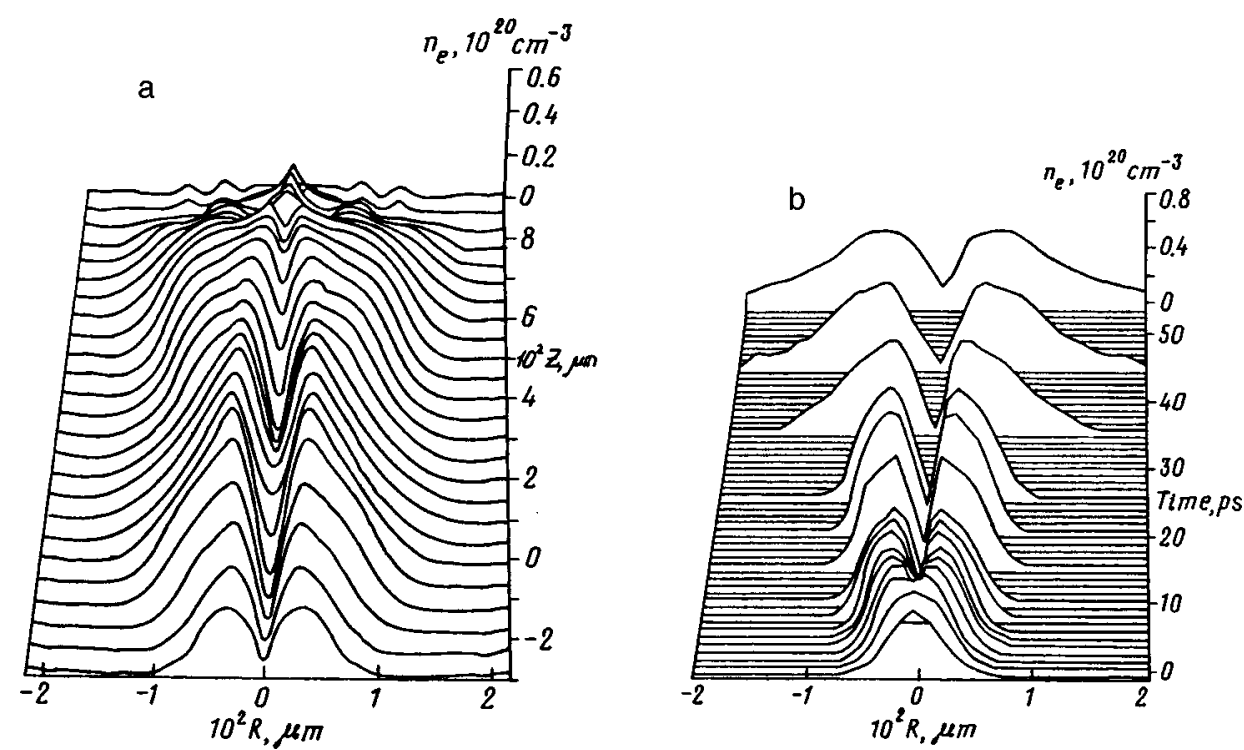

FIG. 2. Two-dimensional electron density distribution, $n_{e}(r, z)$, at time $30 \mathrm{ps}(\mathrm{a})$, and the evolution of the radial electron density profile, $n_{e}(r, t)$, at the axial position $z=100 \mu \mathrm{m}$ from the focus (b).

the focusing conditions and it is about $50 \%$ in the present case. This estimate is deduced from the measurement of the radial energy distribution in the output plane of the laser channel. The actual diameter of the laser channel is probably smaller than the instrumental resolution and was not measured directly in the experiment. (Below we estimate the
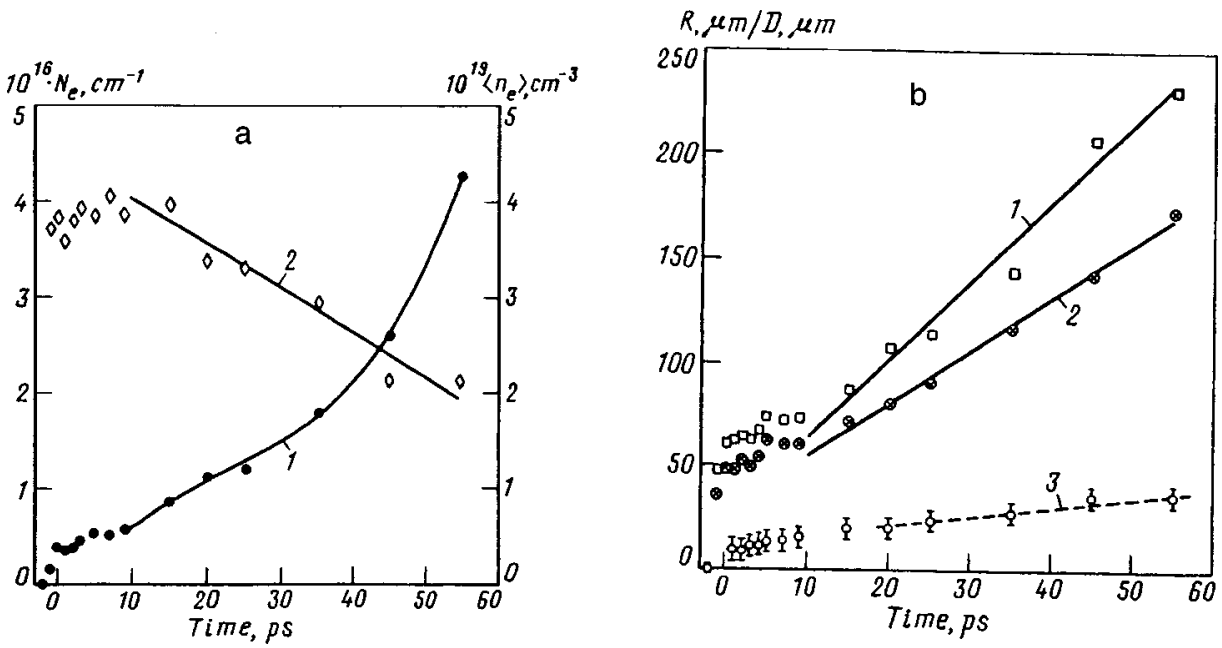

FIG. 3. a: Temporal evolution of the linear electron density, $N_{e}(t)$, (curve 1 ) and the mean electron density (2) in the cross section located at $z=100 \mu \mathrm{m}$ from the focal plane. b: Temporal evolution of the plasma radius at $z=100 \mu \mathrm{m}$ for the $n_{e}=5 \times 10^{18} \mathrm{~cm}^{-3}(1)$ and $1.5 \times 10^{19} \mathrm{~cm}^{-3}$ (2), and the temporal evolution of the plasma channel diameter (3). 
channel radius as $r_{0} \approx 3 \mu \mathrm{m}$ by relating it to the fast ion energy.) The rest of laser power is not trapped and ionizes gas in the laser convergence cone. The measured initial fully ionized plasma radius in the focal plane is $r_{p} \simeq 40 \mu \mathrm{m}$.

The laser pulse exerts a radial ponderomotive force on electrons, expels them from the axis, and forms an electron channel. Its depth, $\delta n_{e} / n_{e}=\lambda_{e}^{2} \nabla_{r}^{2} \sqrt{1+a^{2} / 2}$, is about $10 \%$ for a laser channel radius $r_{0} \approx 3 \mu \mathrm{m}$ and $a^{2} \approx 5$. This formula follows from Poisson's equation and the balance between the electrostatic and ponderomotive potentials. Here $\lambda_{e}=c / \omega_{p}$ is the electron inertia length, $\omega_{p}$ is the electron plasma frequency, and $a=0.85 \times 10^{-9} \lambda[\mu \mathrm{m}] \sqrt{I\left[\mathrm{~W} / \mathrm{cm}^{2}\right]}$ is the dimensionless laser field vector potential in a channel. The ions do not have time to move during the passage of the laser pulse and the electrons return to their original position when the pulse ends. However, the laser pulse supports the electron channel and because of that loses its energy for ion acceleration. Then the ions in the wakee of the laser pulse acquire a kinetic energy and begin to move in radial direction. This effect, known as "'Coulomb explosion," 10 has been discussed as a mechanism of plasma channel formation. ${ }^{4,5}$

For a relatively short laser pulse, $\tau<r_{0} / u_{i} \sim 1 \mathrm{ps}$, the velocity acquired an ion (with the mass $M$ ) from the laser pulse can be estimated as $u_{i}=(Z / M) m c^{2} \nabla_{r} \int d t \sqrt{1+a^{2} / 2}$. Then one finds the ion kinetic energy

$$
\epsilon_{i}=\frac{Z^{2}}{2 M} m^{2} c^{4}\left(\nabla_{r} \int d t \sqrt{1+\frac{a^{2}}{2}}\right)^{2}
$$

and the corresponding pulse energy loss per unit length, $d \mathcal{E} / d z \simeq-2 \pi \int r d r n_{i} \epsilon_{i}$. According to the observations there is a characteristic maximum ion energy $\sim 300 \mathrm{keV}$. Formula (1) also predicts the high-energy cutoff. For estimates we assume a Gaussian pulse shape in time and over the radius, $I=I_{\max } \exp \left(-t^{2} / \tau^{2}-r^{2} / r_{0}^{2}\right)$, with $\tau \simeq 240$ fs. Then to accommodate the maximum ion energy of $300 \mathrm{keV}$ with $50 \%$ laser energy trapping in the channel, the laser channel radius in Eq. (1) has to be $r_{0} \simeq 3 \mu \mathrm{m}$, which determines the maximum laser intensity, $I_{\max } \sim 8.3 \times 10^{18} \mathrm{~W} / \mathrm{cm}^{2}$. These estimates of the channel radius and laser intensity qualitatively agree with the theory of relativistic self-focusing Refs. 1 and 11. The group of fast $\sim 300 \mathrm{keV}$ ions is responsible for the preionization of ambient gas (cf. Fig. 3b, curve 1). Equation (1) predicts a rather wide energy spectrum of the ions with mean energy of $\sim 130 \mathrm{keV}$. These ions are initially concentrated in a cylinder of radius $R \sim 5 \mu \mathrm{m}$ and then begin to expand radially. These ions are responsible for the bulk of the ionization of the ambient gas $(50 \%$, cf. Fig. 3b, curve 2). The characteristic energy deposited into these ions is $d \mathcal{E} / d z \sim 0.5 \mathrm{~J} / \mathrm{cm}$, which constitutes about $6 \%$ of the laser pulse energy trapped in a channel $1 \mathrm{~mm}$ in length.

There are also relatively low-energy ions (about $10 \mathrm{keV}$ ) expelled from a larger radius, $r_{\mathrm{ch}} \approx 15 \mu \mathrm{m}$, which are responsible for the slow dynamics of the plasma channel. They are accelerated by the low-intensity wings of the untrapped part of the laser beam. According to Eq. (1), the intensity required for acceleration of these ions is below $10^{18}$ $\mathrm{W} / \mathrm{cm}^{2}$.

This regime of plasma channel formation due to the ion acceleration ("Coulomb explosion" according to the terminology of Ref. 10) is completely different from the mechanism of plasma thermal heating and electron-impact ionization. In the latter case 
plasma expands with the ion acoustic velocity. ${ }^{2}$ The electron temperature $T_{e}$ can be estimated as about $100 \mathrm{eV}$, according the measurements of Ref. 5 for conditions similar to the present experiment. Therefore the ion acoustic velocity $c_{s}=\sqrt{Z T_{e} / M} \sim 7 \times 10^{6}$ $\mathrm{cm} / \mathrm{s}$ in our experiment is less than one-tenth of the ion expansion velocity. We also disagree with the conclusion of Ref. 5 that the plasma expands at the ion acoustic velocity, since the conditions of that experiment are similar to ours.

Initially the accelerated ions propagate through a plasma $\left(R<r_{p} \sim 40 \mu \mathrm{m}\right)$ and cannot be seen with our diagnostic tools. After a delay time $t_{d}=r_{p} / u_{i} \sim 10$ ps they penetrate into the neutral gas $\left(r>r_{p}\right)$ and begin to ionize it. Although the ionization consumes a negligible part of ion energy, it proceeds very efficiently because the fast ion velocity, $u_{i} \sim(2-4) \times 10^{8} \mathrm{~cm} / \mathrm{s}$, is comparable to the velocity of bound electrons in the helium atom. These conditions correspond to the maximum of the ionization cross-section $\sigma_{i} \sim 3 \times 10^{-16} \mathrm{~cm}^{2}$ (Ref. 13). It is about an order of magnitude larger than the cross section of He ionization due to electron collisions.

The electron density at the radius $r$ behind the group of fast ions can be estimated as $n_{e} \approx n_{H e}^{2} r_{0}^{2} \sigma_{i} / r$. This corresponds to about $10 \%$ ionization at $r \sim 100 \mu \mathrm{m}$. The degree of preionization decreases with $r$ as the fast ion flux decreases. The $100 \mathrm{eV}$ electrons that accompany fast ions can also contribute to the gas ionization at a level of a few percent. According the same formula, the main $(130 \mathrm{keV})$ ions that penetrate into the preionized gas somewhat later produce three times higher ionization at the same distance. Thus one may expect about 50\% ionization for $r \sim 100 \mu \mathrm{m}$, in agreement with experimental results. The total number of the electrons behind the ion front increases linearly with time, while the average electron density is inversely proportional to $t$. All these qualitative relations as well as the number of the electrons agree with data shown in Fig. 3a.

In conclusion, the temporal evolution of the plasma channel created due to the relativistic self-focusing of a $4 \mathrm{TW}, 400 \mathrm{fs}$ laser pulse has been observed for the first time using the interferometric technique with high spatial and temporal resolution. By comparison of the experimental data with theoretical estimates we demonstrate that the channel evolution is dominated by the Coulomb explosion effect, with the subsequent penetration of high-energy ions into the ambient neutral gas. This experiment reveals a new efficient mechanism of direct deposition of the laser energy into the high-energy ions ( $\sim 6 \%$ for our conditions), which can play a fundamental role in the absorption short, high-intensity laser pulses. We estimate the fast ion energy as $\sim 300 \mathrm{keV}$ or $80 \mathrm{keV} /$ nucleon, which is comparable to that observed in a solid-target experiment. ${ }^{14}$

The authors would like to acknowledge Dr. S. Pikuz for useful discussion. This work was supported by NSF PHY 972661, NSF STC PHY 8920108, DOE/LLNL subcontract B307953, and the Russian Fund for Fundamental Research (Grants 96-0216707-a and 96-02-16165-a).

a)e-mail: sarkisov@sci.lebedev.ru

\footnotetext{
${ }^{1}$ A. B. Borisov, A. V. Borovskiy, V. V. Korobkin et al., Phys. Rev. Lett. 68, 2309 (1992).

${ }^{2}$ M. Dunne, T. Afshar-Rad, J. Edwards et al., Phys. Rev. Lett. 72, 1024 (1994).

${ }_{3}^{3}$ A. J. MacKinnon, M. Borgesi, A. Iwase et al., Phys. Rev. Lett. 76, 1473 (1996); M. Borghesi,

A. J. MacKinnon, L. Barringer et al., Phys. Rev. Lett. 78, 879 (1997).
} 
${ }^{4}$ D. Umstadter, S. Y. Chen, A. Maksimchuk et al., Science 273, 472 (1996).

${ }^{5}$ K. Krushelnik, A. Ting, C. I. Moore et al., Phys. Rev. Lett. 78, 4047 (1997).

${ }^{6}$ V. Malka, E. Wispelaere, F. Amiranoff, and S. Baton, Phys. Rev. Lett. 79, 2979 (1997).

${ }^{7}$ P. Maine, D. Strickland, P. Bado et al., IEEE J. Quantum Electron. QE-24, 398 (1988); M. D. Perry and G. Mourou, Science 264, 917 (1994).

${ }^{8}$ G. S. Sarkisov, Instrum. Exp. Tech. 39, 727 (1996).

${ }^{9}$ S. Augst, D. Strickland, D. D. Meyerhofer et al., Phys. Rev. Lett. 63, 2212 (1989).

${ }^{10}$ N. H. Burnett and G. D. Enright, IEEE J. Quantum Electron. QE-26, 1797 (1990).

${ }^{11}$ P. Sprangle, E. Esarey, J. Krall, G. Joyce, Phys. Rev. Lett. 69, 2200 (1992); K. C. Tzeng, W. B. Mori, C. D. Decker, Phys. Rev. Lett. 76, 332 (1996); P. Mora and T. M. Antonsen, Phys. Plasmas 4, 217 (1997).

${ }^{12}$ T. E. Glover, T. D. Donnelly, E. A. Lipman et al., Phys. Rev. Lett. 73, 78 (1994); W. J. Blyth, S. G. Preston, A. A. Offenberger et al., Phys. Rev. Lett. 74, 554 (1995).

${ }^{13}$ R. K. Janev, L. P. Presnyakov, and V. P. Shevelko, in Physics of Highly Charged Ions, edited by G. Ecker, Springer-Verlag, 1985.

${ }^{14}$ A. Fews, P. A. Norreys, F. N. Beg et al., Phys. Rev. Lett. 73, 1801 (1994).

Published in English in the original Russian journal. Edited by Steve Torstveit. 